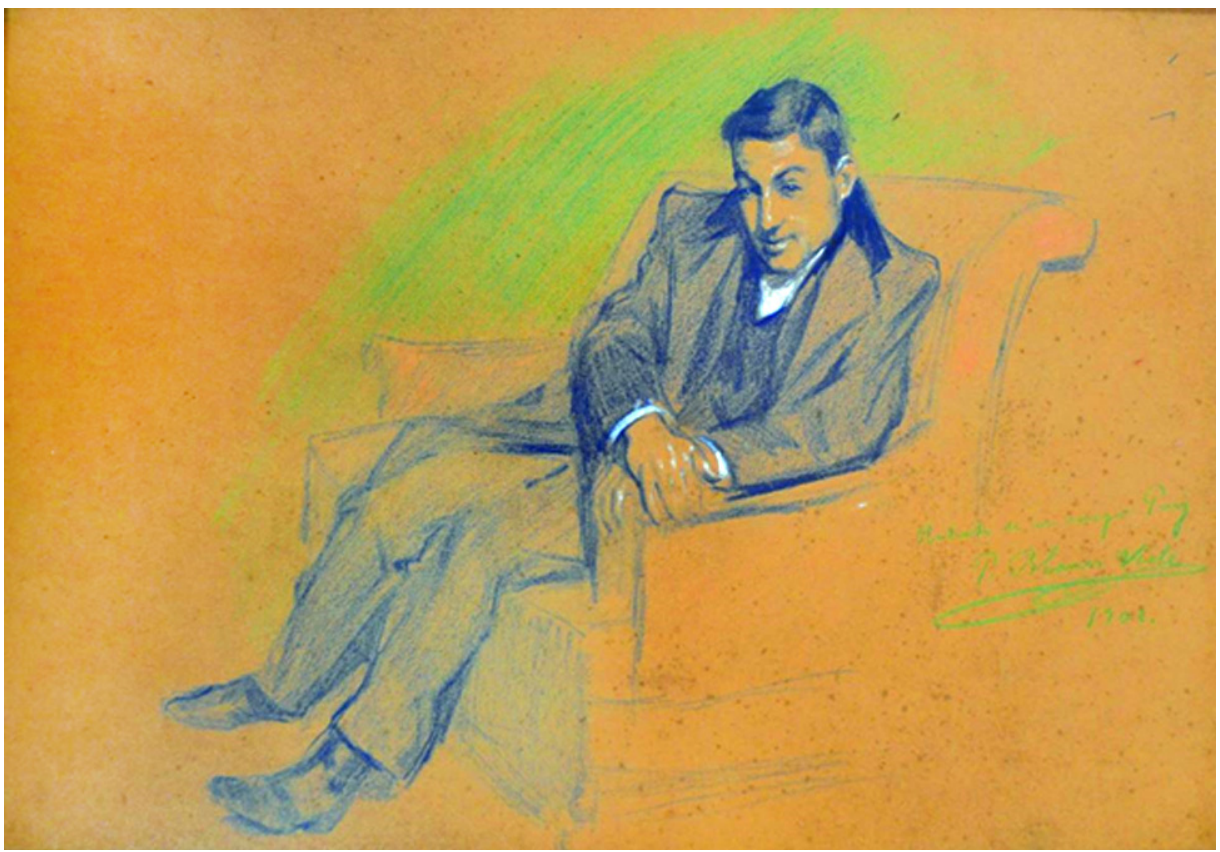

Pedro Blanes Viale ( 1878 - 1926), "Retrato de Vicente Puig", técnica mixta sobre papel (1902), titulado por el artista "Retrato de mi amigo Puig".

\title{
Disyuntivas económicas y políticas de la Guerra Fría en América Latina
}

Resumen: En este artículo se hace una sintética reflexión sobre cómo América Latina afrontó los "desafíos del desarrollo económico" entre 1959 y 1989 en unas décadas enmarcadas en el escenario político y social de revoluciones, dictaduras y democracias. El eje del trabajo está puesto en cómo se fueron implementando diferentes políticas económicas en el marco de un escenario político cambiante en cada década.

Palabras clave: Guerra fría - Latinoamérica- Democracia - Revolución - Desarrollo Económico 
Los treinta años que transcurrieron desde 1959 hasta 1989 fueron cruciales para la historia reciente latinoamericana: fue una era de revoluciones, dictaduras, democracia y reformas económicas que dieron forma a una etapa compleja de consignas, ideologías, violencia, pero también esperanzas que pretendieron reemplazar las viejas estructuras heredadas del pasado.

Para algunos la entrada de Fidel Castro el $1^{\circ}$ de enero de 1959 a La Habana y la figura del argentino Ernesto «che» Guevara se convirtieron en los líderes a quienes seguir. Otros se opusieron a ese liderazgo revolucionario y antidemocrático apoyando gobiernos militares también denominados burocrático-autoritarios o simplemente dictaduras. ${ }^{1}$ En tanto que unos terceros, en la búsqueda de soluciones económicas que aliviaran las tensiones, miraron las propuestas de desarrollo que hizo la Comisión Económica para América Latina (CEPAL) creada en 1948 y cuya sede se encuentra en
Santiago de Chile. Su objetivo era promover el desarrollo económico y social mediante la cooperación e integración regional y subregional, entendiendo que el continente había emprendido una transformación de las estructuras económicas basada en el keynesianismo. Como parte de la división internacional del trabajo consideró a Latinoamérica como parte de la periferia y productor de materias primas y alimentos que abastecían los centros industriales. Un modelo «centro periferia» que transfería desigualmente la innovación técnica y la utilidades, provocando el deterioro de los términos de intercambio. ${ }^{2}$

Fueron años de tensiones y rupturas de consensos. El enemigo a quien debían enfrentar los países pasó de ser externo, es decir estar fuera de las fronteras nacionales, a estar al interior de cada país con lo que se desataron conflictos entre los propios connacionales que en muchos casos hicieron que la política se convirtiera en un juego suma cero que fracturó la democracia y dividió a la población. En un

1 Guillermo O’ Donnell, 1966-1973: El Estado burocrático autoritario: triunfos, derrotas y crisis (Buenos Aires: Belgrano, 1982).

2 Véase Hernán Santa Cruz, "La creación de las Naciones Unidas y de la CEPAL", Revista de la CEPAL, n 57 (diciembre de 1995): 25; Fernando Cardoso, Aníbal Pinto y Osvaldo Sunkel (dirs.), El pensamiento de la CEPAL (Santiago: Editorial Universitaria, 1969), 15-20; Carlos Malamud, Historia de América (Madrid: Alianza Editorial, 2005), 417; Rosemary Thorp, Progreso, pobreza y exclusión: Una bistoria económica de América Latina en el siglo XX (Washington DC: BID, 1998), 141-142; Carlos Sabino, El fracaso del intervencionismo: Apertura y libre mercado en América Latina (Santiago: Democracia y Mercado, 2012) y Alejandro San Francisco, et al., Historia de Chile 1960-2010. Tomo 1. Democracia, esperanzas y frustraciones. Chile a mediados del siglo XX (Santiago: CEUSS, 2016), 214. 
mundo fraccionado artificialmente entre capitalismo y comunismo con dos esferas de influencia como Estados Unidos y la Unión de Repúblicas Socialistas Soviéticas, las superpotencias pretendieron apropiarse de lo que se llamó «sus áreas de influencia». En el caso de América Latina, Estados Unidos si bien le prestaba poca atención, siempre consideró que esta le pertenecía e incluso para algunos era considerada su «patio trasero». Rosemay Thorp señala al respecto: «La baja prioridad que acordaba Estados Unidos a América Latina se haría evidente con el tiempo. Como región, era considerada relativamente 'segura', aunque se pensaba que era necesario ayudarla a controlar los movimientos obreros. La batalla ideológica mundial fue tan importante en la región como en cualquier otro sitio durante esos años, y había que vigilar estrechamente la 'subversión comunista'». El problema fue que no se contempló una ayuda material concreta - a excepción de la fallida Alianza para el Progreso- al estilo de un Plan Marshall, ya que como continúa esta misma autora, era mucho más esencial otras regiones como Europa y Asia. ${ }^{3}$
Pero el temor estadounidense no era infundado, a lo largo de los años sesenta Fidel Castro apoyó los movimientos guerrilleros en Guatemala, Nicaragua, Venezuela, Colombia, Perú, Argentina, Bolivia y Chile. Esto significó que la eventual caída de la región en las manos del comunismo, especialmente tras las revoluciones de Bolivia en 1952, Guatemala en 1954 y Cuba en 1959, alertó a los norteamericanos quienes presionaron para «frenar los movimientos reformistas de cualquier tipo que pudieran identificarse con la izquierda». ${ }^{4} \mathrm{El}$ temor a que de manera secuencial los países fueran cayendo bajo la órbita soviética, hizo que se acuñara la «teoría del dominó» que se trató de evitar, primero ayudando política y económicamente con las propuestas de la Alianza para el Progreso (1961), pero luego -fundamentalmente- a través de asistencia y entrenamiento militar a los ejércitos nacionales dando origen a la Doctrina de Seguridad Nacional. ${ }^{5}$

Alan Angell señala que «el triunfo de la revolución cubana quitó validez a la pretensión de los partidos comunistas ortodoxos de ser la única fuente de legitimidad marxista $y$,

\footnotetext{
3 Thorp, Progreso, pobreza y exclusión, 138.

4 Alan Angell, "La izquierda en América Latina desde 1920", en Historia de América Latina. 12 Política y sociedad desde 1930, ed. Leslie Bethell (Crítica: Barcelona, 1997), 100.

5 Pedro Rivas Nieto, Doctrina de seguridad nacional y regímenes militares en Iberoamérica (San Vicente/Alicante: Editorial Club Universitario, D.L., 2008).
} 
por ende, revolucionaria». Agrega: «muchos radicales jóvenes pensaban que el entusiasmo y el compromiso eran suficientes para hacer la revolución. La mayoría de los aspirantes a imitar a Castro abogaban por la guerra de guerrillas, pero incluso los que no opinaban igual eran partidarios de un radicalismo político que derribase las estructuras existentes». ${ }^{6}$

El golpe de Estado de 1964 en Brasil, si bien confirmó la fragilidad democrática latinoamericana que durante el siglo XX se debatió entre democracia, intervenciones militares, dictaduras y democracia, fue el primero en que los militares actuaron como institución. ${ }^{7} \mathrm{Ya}$ no era un caudillo que levantó a sus compañeros de armas y dio un cuartelazo. Su objetivo era cambiar el sistema político y económico. Jonathan Hartlyn y Arturo Valenzuela, señalan que «al hacerse con el poder, los militares brasileños demostraron desprecio por la política democrática paralizada y los llamamientos populistas, a la vez que ponían en práctica una política represiva contra la izquierda revolucionaria». Agregan que «el golpe brasileño señaló un cambio cualitativo en el gobierno de los militares en el continente e instauró un gobierno que no se consideraba a sí mismo sencillamente como árbitro, sino como fuerza revolucionaria que pretendía forjar un nuevo orden político y económico muy diferente al modelo cubano». ${ }^{8}$

En Argentina las intervenciones militares fueron en 1966 y 1976; en Perú 1968, Bolivia 1971, Ecuador 1972 y Chile y Uruguay en 1973.

Es decir, incubado en los cincuenta y durante la década del sesenta, estuvo presente la sensación que «todos querían la revolución», entendiendo por tal la necesidad de un cambio profundo en las estructuras dominantes en Latinoamérica. Lo anterior significó que al finalizar los años setenta, solo Costa Rica, Colombia y Venezuela tenían sistemas democráticos, aunque en el caso colombiano, siempre estaba amenazada por la creciente presencia del narcotráfico y las guerrillas rurales. ${ }^{9}$ México, era un caso especial, controlado por el Partido Revolucionario Institucional (PRI), se había convertido en lo que Mario Vargas Llosa llamó en 1990: «la dictadura perfecta» a lo que el poeta Octavio Paz

6 Alan Angell, "La izquierda en América Latina desde 1920”, 100.

7 Angel Soto y Paula Schmidt (ed.), Las frágiles democracias latinoamericanas (Santiago: Aguilar-El Mercurio, 2008).

8 Jonathan Hartlyn y Arturo Valenzuela, “La democracia en América Latina desde 1930”, en Leslie Bethell, (ed.), Historia de América Latina. 12 Política y sociedad, desde 1930 (Barcelona: Crítica, 1997), 49.

9 Hartlyn y Valenzuela, "La democracia en América Latina desde 1930", 50. 
corrigió denominando «un sistema hegemónico de dominación»». ${ }^{10}$

En los años ochenta, los militares comenzaron a retirarse del poder. El primero fue Perú en 1980, luego Argentina en 1983, Uruguay en 1984, Brasil en 1985 y finalmente Chile en $1990 .{ }^{11}$ A partir de entonces, se produjo una doble transición a la democracia o «tercera ola democratizadora» en el mundo. ${ }^{12}$ Por un lado, estaba el paso desde las dictaduras a regímenes elegidos por los ciudadanos los que fueron echando raíces permitiendo avanzar hacia la gobernabilidad y la consolidación democrática. Pero, por otro lado, también se produjo un cambio en el modelo de desarrollo imperante que estaba agotado para 1970 y que tras la crisis de la deuda a comienzos de los ochenta, pasó desde una economía de Industrialización Sustitutiva de Importaciones (ISI) puesta en marcha a fines de los años treinta y continuada en parte con las propuestas cepalianas, las cuales básicamente plantearon un protagonismo del Estado con un desarrollo hacia adentro con políticas proteccionistas y economías parcialmente cerradas, hacia un modelo de apertura económica y libre mercado centrado en el individuo y la privatización de las empresas públicas. Se tuvo a la vista la exitosa recuperación económica chilena, tras la fallida experiencia de la Unidad Popular, que desde 1975 había sembrado las bases para que desde mediados de los ochenta y hasta 1997 tuviera un crecimiento sostenido del PIB en torno al 7\% promedio. $^{13}$

Los ochenta son recordados como una "década perdida» en materia económica. La crisis del petróleo hizo que Europa y Estados Unidos sufrieran un proceso de estancamiento e inflación que hizo caer la demanda afectando a los países latinoamericanos productores de materias primas quienes desde mediados de los setenta - con la anterior crisis- se habían comenzado a endeudar fuertemente. La deuda externa latinoamericana pasó de un $152 \%$ de las exportaciones en 1980 a $241 \%$ en 1982 , en tanto que el

10 "Vargas Llosa: 'México es la dictadura perfecta”, El País (España), 1 de septiembre, 1990. https://elpais.com/ diario/1990/09/01/cultura/652140001_850215.html (Consultado 4 de marzo de 2020).

11 Hartlyn y Valenzuela, "La democracia en América Latina desde 1930”, 50.

12 Huntington Samuel, The Third Wave: Democratization in the Late Twentieth Century (Oklahoma: University of Oklahoma Press, 1991).

13 Véase Thorp, Progreso, pobreza y exclusión, 1998; Raúl Sanhueza y Angel Soto, "Un proyecto para América Latina: el consenso de Chile”, en Pedro Isern y Gabriel Salvia (comp.), La experiencia chilena: Consensos para el desarrollo (Buenos Aires: Cadal, 2005), 151-178; Sebastián Edwards, Crisis y reforma en América Latina: Del desconsuelo a la esperanza (Buenos Aires: Emecé, 1995); Sebastián Edwards, Populismo o mercado: El dilema de América Latina (Bogotá: Norma, 2009) y Carlos Sabino, El fracaso del intervencionismo. 
precio de las materias primas cayó $11 \%$ en los mismos años. La subida de las tasas de interés por parte del gobierno estadounidense hizo estallar la crisis cuando México se declaró en moratoria respecto del pago de la deuda. ${ }^{14}$

La necesidad de colaborar entre los países trajo de regreso el viejo integracionismo latinoamericano, que tuvo como resultado la creación en 1990 del Mercado Común del Sur (MERCOSUR).

Pero la crisis y el debilitamiento de las dictaduras, permitió que se fueron recuperando ciertos consensos que se habían quebrado en el pasado. El más importante es que las transiciones a la democracia fueron el resultado de acuerdos entre los militares y civiles, con excepción de Argentina cuyo régimen militar colapsó tras la derrota en la guerra de Las Malvinas (1982), el golpe de Estado en Paraguay que acabó con la dictadura de Alfredo Stroessner (1989) o en Panamá tras la invasión norteamericana en 1989. Algo similar puede decirse de El Salvador en 1979 y Nicaragua en 1990.

Las últimas batallas de la guerra fría en Latinoamérica se dieron en Centroamérica, convirtiendo a la zona en un foco de tensión durante los ochenta. El debilitamiento de los gobiernos militares y el triunfo sandinista en Nicaragua en 1979 apoyado desde Cuba y la URSS provocaron una guerra civil que los enfrentó con la Contra apoyada por Estados Unidos y Honduras, pero también reactivaron la lucha guerrillera en Guatemala, mientras que en El Salvador surgió el Frente Farabundo Martín para la Liberación Nacional (FMLN).

La caída del Muro de Berlín en 1989 con la consiguiente disolución de la URSS en 1991, así como la mediación de Óscar Arias en los procesos de paz en Centroamérica, lo que le valió el Premio Nobel de la Paz en 1987, pusieron «fin» a la guerra fría en Latinoamérica. Sin embargo, la historia no terminaría. Tras unos años noventa donde convivió la libertad política con la libertad económica haciendo pensar que finalmente la región había encontrado el camino para su «despegue», el término del siglo XX evidenció que faltaba mucho que avanzar. Un ejemplo son las posibilidades de involución autoritaria que se creían superadas en los noventa, época en que se pensó que no se volvería a tocar las puertas de los cuarteles para resolver los problemas políticos, quedaron en

14 Manuel Alcántara, et. al., Historia de América latina v. 6: Reformas económicas y consolidación democrática (1980-2006) (Madrid: Síntesis, 2006), 14. Véase también Thorp, Progreso, pobreza y exclusión, 1998, 232-237. Edwards, Populismo o mercado, 2009 y José A. Ocampo, et. al., La crisis latinoamericana de la deuda desde la perspectiva bistórica (Santiago: CEPAL, 2014). 
entredicho con la llegada al poder de Hugo Chávez en Venezuela en 1998. Pero, independiente del caso venezolano, las tensiones no han terminado ya que prevalecen la inestabilidad política y la desigualdad económica que impiden superar las crisis de gobernabilidad recurrentes en el continente. ${ }^{15}$

La presencia permanente de caudillos populistas hace que la región no logre superar ese vicio del pasado ni que las instituciones funcionen, manteniendo -como dice el historiador Carlos Malamud«los tópicos de ayer, de hoy y de siempre». ${ }^{16}$ Tampoco en la región está superado del todo la dicotomía ideológica de la guerra fría: capitalismo o socialismo, en estas dos primeras décadas del siglo XXI el debate, la tensión y -más recientemente- la violencia han regresado con algunas consignas muy similares a las de los años sesenta.

En marzo del 2019, un artículo publicado en CNN se preguntaba si acaso estaba «¿de vuelta la guerra fría?», en alusión a los problemas de violencia que vive Centroamérica donde el «deshielo» no llegó. ${ }^{17}$ Las consignas de las protestas de ese año que continúan hasta el presente hacen pensar que no es una preocupación exclusiva de esa zona, sino de todo el continente.

A nivel mundial hay quienes ya hablan de una «segunda guerra fría», mientras que autores como Robert Kagan afirman que las tensiones entre las potencias hacen que el mundo se ha vuelto «otra vez normal». ${ }^{18}$ En ese contexto, Latinoamérica seguirá debatiéndose y mirando a otros centros de poder, pero con las mismas carencias y tensiones que la afectaron durante la guerra fría.

Con los antecedentes antes expuestos, a continuación, se hace una breve presentación de cada uno de los artículos contenidos en el monográfico.

Hugo Harvey en su artículo aborda la crisis de República Dominicana en 1965, a la que considera «el» punto de inflexión del sistema interamericano durante la Guerra Fría por la utilización de la Organización de Estados Americanos (OEA) a los fines

15 Manuel Alcántara, et. al., Historia de América latina v. 6.

16 Carlos Malamud, Populismos latinoamericanos: Los tópicos de ayer, de hoy y de siempre (Oviedo: Ediciones Nobel, 2010). 17 Vaclav Masek Sánchez, "De vuelta a la guerra fría? Centroamérica se vuelve a congelar", CNN, 25 de marzo de 2019. https: / cnnespanol.cnn.com/2019/03/25/de-vuelta-a-la-guerra-fria-centroamerica-se-vuelve-a-congelar/ (Consultado 4 de marzo de 2020).

18 Véase: Mary Kaldor, "La segunda guerra fría”, El País (España), 13 de marzo, 2016. https://elpais.com/ internacional/2016/03/09/actualidad/1457541642_608147.html (Consultado 4 de marzo de 2020); Robert Kagan, The Return of History and the End of Dreams (Nueva York: Vintage Editions, 2008). 
políticos de Estados Unidos para legitimar su invasión con una fuerza interamericana de paz. En su investigación se reconstruye el proceso de invasión estadounidense en República Dominicana desde fuentes primarias de actores políticos de la época y de las distintas posiciones historiográficas sobre el acontecimiento. Un aporte valioso de su trabajo es cómo esto incidió en la OEA mostrando discusiones entre los representantes de los países miembros para avalar la constitución de la fuerza interamericana de paz. A partir de allí reconstruye el rechazo que este hecho tuvo entre las elites políticas, los movimientos estudiantiles y sindicales, además de la repercusión mediática en los países latinoamericanos. Además, dedica espació a la incidencia de este acontecimiento en el mundo de las artes y las letras. Las críticas no solo se centraron en Estados Unidos, sino en el accionar de la OEA, de la CEPAL y hasta de la ONU. Muestra también cómo este acontecimiento desencadenó repercusiones globales, de hecho, el Consejo de Seguridad de Naciones Unidas mantuvo veintiocho reuniones sobre la crisis. La originalidad de su artículo radica en mostrar cómo en el 2015 al cumplirse el cincuenta aniversario no hubo análisis político ni historiográficos disparadores para la reflexión histórica y de memoria. Además, reconstruye

como en el cuadragésimo sexto período ordinario de sesiones de la Organización de Estados Americanos celebrado en Santo Domingo, el presidente dominicano, Danilo Medina, tuvo oportunidad de alzar su voz para condenar la violación de su soberanía interna. $\mathrm{El}$ autor, asimismo, logra insertar el acontecimiento con el escenario de la guerra fría global, y en concreto, realiza una serie de conexiones con la guerra de Vietnam, que le permiten desde fuentes desclasificadas de la CIA y de documentación secundaria poner en discusión las razones que tuvo Lyndon B. Johnson para intervenir en República Dominicana.

El artículo de José Antonio Ramírez Díaz hace un exhaustivo análisis historiográfico sobre el valor estratégico que se dio a la ciencia en la construcción del mundo de la segunda posguerra, y en concreto sobre cómo Estados Unidos colaboró en la institucionalización de los Consejos de Ciencia y Tecnología en distintos países de América Latina en las décadas de los sesenta y setenta. El artículo muestra, desde una bibliografía exhaustiva y fuentes documentales de distintos organismos internaciones y de otros programas, cómo Estados Unidos aprovechó su liderazgo para influir en el armado de estrategias de colaboración científica para la elaboración de agendas 
económicas y aplicación de políticas para combatir el subdesarrollo y la problemática de la pobreza en los países latinoamericanos. Conecta principios y objetivos sobre ciencia y desarrollo, contenidos en la ONU, UNESCO, Banco Interamericano de Desarrollo, CEPAL, Alianza para el Progreso y fundaciones privadas estadounidenses, para mostrar las influencias que ejercieron en el proceso de institucionalización de la ciencia en América Latina. En su investigación se visualiza cómo Estados Unidos usó la investigación científica institucionalizada como un mecanismo para contrarrestar el avance de su enemigo comunista en la región.

Ivan Witker en su artículo, «Alemania oriental y América Latina durante la Guerra Fría: trazos geopolíticos y resiliencia cultural», parte de considerar cómo la República Democrática Alemana era en 1990 el régimen comunista de mejores resultados económicos y sociales, al tiempo que destaca a dos de sus dirigentes, Erich Honecker y Werner Lamberz como los principales arquitectos de la construcción del nuevo reposicionamiento de la política exterior hacia América Latina. Además de ser forjadores de lo que se llamó «sociedad socialista desarrollada» como «matriz diferenciadora del resto de los países del bloque soviético». El aporte medular de su trabajo lo constituyen las relaciones que entabló la RDA con el gobierno de Salvador Allende. Así señala que la embajada germano oriental en Santiago funcionó como «punta de lanza» para su ofensiva diplomática en América Latina. Producido el golpe de Estado de Augusto Pinochet, la RDA acogió a exiliados chilenos, y Witker muestra cómo estos jugaron un rol protagónico cuando se dio el retorno de la democracia en Chile para acoger a Honecker y a su mujer como refugiados ante la persecución judicial alemana. Witker lo define como una "deuda inserta en un espiral de fidelidades». El punto cardinal de su artículo, y que aporta valiosas lecturas y discusiones de la historiografía alemana, es cómo la RDA «forjó y proyectó una identidad que sobrepasaba con creces sus capacidades geopolíticas», lo que explica, en parte, la existencia de la Ostalgie.

Por último, la contribución de Héctor Ghiretti incide en uno de los aspectos más interesantes menos conocidos de la Guerra Fría en el «teatro de operaciones» América Latina: la presencia y las actividades de los servicios de inteligencia del Bloque Oriental en la región. A partir de las revelaciones en torno a las vinculaciones de Vivian Trías, destacado intelectual y dirigente 
del Partido Socialista Uruguayo, con la inteligencia checoslovaca, se abre una gama de problemas historiográficos relacionados con el compromiso político y la militancia en organizaciones revolucionarias. En particular, el autor analiza el problema de la proporcionalidad de los medios respecto de los fines perseguidos. Las modalidades de la lucha revolucionaria exceden la participación en la esfera pública y los canales de la representación política, el proselitismo y la organización partidaria. El episodio debe contextualizarse en la prolongada crisis e impugnación del liberalismo, que inició en los años del período de entreguerras y que se cerraría varias décadas después de la Segunda Guerra Mundial. El cese de la confrontación bipolar pondría un fin provisorio a esta larga crisis.

A modo de conclusión, el dossier monográfico contiene investigaciones que abordan temáticas cardinales que cruzan la guerra fría en América Latina, ya producida la revolución cubana, y complementan está introducción centrada en las «Disyuntivas económicas» que atravesó la región. Una de las temáticas es el papel de los Estados Unidos y su influencia en la región, ya sea directa o por medio de organismos internacionales, como es el caso de los trabajos de Hugo Harvey y de José Antonio
Ramírez Díaz. Asimismo, el estudio de la guerra fría no puede dejar de contemplar a otro de sus contrincantes: el comunismo, en ello el trabajo de Ivan Witker sobre la República Democrática Alemana es un aporte original a la complejidad de relaciones que se entablaron con el bloque soviético; y el de Héctor Ghiretti que a partir del intelectual socialista uruguayo Vivián Trías muestra la agenda de discusión de las izquierdas latinoamericanas en aquel tiempo convulso y las repercusiones del caso en el presente uruguayo.

\section{Bibliografía}

Alcántara, Manuel, et al. Historia de América latina v. 6: Reformas económicas y consolidación democrática (1980-2006). Madrid: Síntesis, 2006.

Bethell, Leslie, ed. Historia de América Latina. 12 Política y sociedad desde 1930. Barcelona: Crítica, 1997.

Cardoso, Fernando, Aníbal Pinto, y Osvaldo Sunkel, dirs. El pensamiento de la CEPAL, Santiago: Editorial Universitaria, 1969.

Edwards, Sebastián. Populismo o mercado. El dilema de América Latina. Bogotá: Norma, 2009. 
Edwards, Sebastián. Crisis y reforma en América Latina: Del desconsuelo a la esperanza. Buenos Aires: Emecé, 1995.

Huntington, Samuel. The Third Wave: Democratization in the Late Twentieth Century. Oklahoma: University of Oklahoma Press, 1991.

Kagan, Robert. The Return of History and the End of Dreams. Nueva York: Alfred a Knopf, 2008.

Malamud, Carlos, Populismos latinoamericano: Los tópicos de ayer, de boy $y$ de siempre. Oviedo: Ediciones Nobel, 2010.

Malamud, Carlos. Historia de América. Madrid: Alianza Editorial, 2005.

Ocampo, José A., et al. La crisis latinoamericana de la deuda desde la perspectiva histórica. Santiago: CEPAL, 2014.

O’ Donnell, Guillermo. 1966-1973: el Estado burocrático autoritario: triunfos, derrotas y crisis. Buenos Aires: Editorial de Belgrano, 1982.

Rivas Nieto, Pedro. Doctrina de seguridad nacional y regimenes militares en Iberoamérica. San Vicente (Ali- cante): Editorial Club Universitario, D.L., 2008.

Sabino, Carlos. El fracaso del intervencionismo: Apertura y libre mercado en América Latina. Santiago: Democracia y Mercado, 2012.

San Francisco, Alejandro, et. al. Historia de Chile 1960-2010. Tomo 1. Democracia, esperanzas y frustraciones: Chile a mediados del siglo XX. Santiago: CEUSS, 2016.

Sanhueza, Raúl y Ángel Soto. "Un proyecto para América Latina: el consenso de Chile", en La experiencia chilena: Consensos para el desarrollo, compilado por Pedro Isern y Gabriel Salvia, 151-178. Buenos Aires: Cadal, 2005.

Santa Cruz, Hernán. "La creación de las Naciones Unidas y de la CEPAL". Revista de la CEPAL, $\mathrm{n}^{\circ} 57$ (diciembre de 1995).

Soto, Angel y Paula Schmidt, ed. Las frágiles democracias latinoamericanas. Santiago: Aguilar / El Mercurio, 2008.

Thorp, Rosemary. Progreso, pobreza y exclusión. Una historia económica de América Latina en el siglo XX. Washington DC: BID, 1998. 
Ángel Soto

Universidad de los Andes, Chile angelsoto@uandes.cl

ORCID: http://orcid.org/0000-0003-2769-0163

Web of Science Research ID: K-1680-2016

Carolina Cerrano

Universidad de Montevideo, Uruguay

ccerrano@um.edu.uy

ORCID: http://orcid.org/0000-0002-054I-9623

Para citar este artículo / To reference this article / Para citar este artigo

Soto, Ángel y Carolina Cerrano. "Disyuntivas económicas y políticas de la guerra fría en América Latina". Humanidades: revista de la Universidad de Montevideo, nº 7 (2020): 9-20. https://doi.org//0.25185/7.1

\section{Economic and political dilemmas of the Cold War in Latin America}

Abstract: In this article, a synthetic reflection is made on how Latin America faced the "challenges of economic development" between 1959 and 1989 in a few decades framed in the political and social scenario of revolutions, dictatorships and democracies. The focus of the work is on how different economic policies were implemented within the framework of a changing political scenario in each decade.

Key words: Cold war - Latin America - Democracy - Revolution- Economic Development

\section{Disjuntivas económicas e políticas da Guerra Fria em América Latina}

Resumo: Nesse artigo, tenta-se refletir sobre como a América Latina enfrentou os "desafios do desenvolvimento econômico" entre 1959 e 1989, durante décadas enquadrada como um centro não político e social para revolucionários, cidades e democracias. 0 exo do trabhalo e focado em como as diferentes políticas econômicas são implementadas, e não a estrutura de um censo político em mudança a cada década.

Palavras chave: Guerra Fria - América Latina- Democracia - Revolução - Desenvolvimento Econômico 